\title{
Impact of Propranolol on Preventing Renal Dysfunction in Patients with Cirrhosis
}

\author{
Kambiz Akhavan Rezayat ${ }^{1}$, Abbas Ali Zeraati ${ }^{2, *}$, Masoud Pezeshki Rad ${ }^{3}$, Jalal Chogan ${ }^{1}$, \\ Najmeh Davoudian ${ }^{1}$, Amir Akhavan Rezayat ${ }^{4}$, Seyed Mousalreza Hoseini ${ }^{5}$
}

1. Gastroenterology and Hepatology Research Center, Mashhad University of Medical Sciences, Imam Reza Hospital, Mashhad, Iran

2. Associate Professor of Nephrology, Kidney Transplantation Complications Research Center, Imam Reza Hospital, Mashhad University of Medical Sciences, Mashhad, Iran

3. Department of Radiology, Imam Reza Hospital, School of Medicine, Mashhad University of Medical Sciences, Mashhad, Iran

4. Student Research Committee, Faculty of Medicine, Mashhad University of Medical Sciences, Mashhad, Iran

5. Department of Internal Medicine, School of Medicine, Mashhad University of Medical Sciences, Mashhad, Iran

\section{* Corresponding Author:}

Abbas Ali Zeraati, MD

Kidney Transplantation Complications Research Center, Imam Reza Hospital, Mashhad University of Medical Sciences, Mashhad, Iran

Telefax: + 985138598818

Email: zeraatiA@mums.ac.ir

Received: 01 May 2017

Accepted: 02 Sep. 2017

\section{ABSTRACT}

\section{BACKGROUND}

One of the earliest diagnostic signs of hepatorenal syndrome in patients suffering from liver cirrhosis is an increase in the renal vascular resistive index (RI). In this study, the impact of propranolol on decreasing this index and to postpone the probability of hepatorenal syndrome has been investigated.

\section{METHODS}

In the current research, 30 patients with liver cirrhosis with different age and sexes have been enrolled. Demographic data and complete medical history have been collected using a specific questionnaire. At first, renal artery Doppler ultrasonography was performed to determine the RI. The patients were then treated with propranolol, and under supervision, the dose of the drug was increased gradually every 3 to 5 days to reach the target of $25 \%$ decrease in resting heart rate. One month after reaching the target dose of the medicine, Doppler ultrasonography was repeated for the patients and the second RI was compared with the pretreatment ones.

\section{RESULTS}

According to our results after treatment with propranolol, a significant decrease of RI was observed $(p<0.01)$. However, there was no significant difference in the glomerular filtration rate (GFR) before and after treatment with propranolol $(p=0.290)$. In our study, we found that administering propranolol was associated with significant changes in RI and GFR between the patients with compensated and decompensated cirrhosis (mean change: $-0.005 \pm 0.017$ vs. $-0.058 \pm 0.045$; $p<0.01$ for RI and $-4.226 \pm 17.440$ vs. $13.486 \pm 12.047 ; p<0.01$ for GFR in patients with compensated and decompensated cirrhosis, respectively).

\section{CONCLUSION}

Propranolol reduces renal vascular RI in patients with cirrhosis. The response rates in the patients with decompensating cirrhosis were significantly higher than the patients with compensating cirrhosis

\section{KEYWORDS:}

Renal vascular resistive index, Cirrhosis, Color Doppler ultrasonography

Please cite this paper as:

Akhavan Rezayat K, Zeraati AA, Pezeshki Rad M, Chogan J, Davoudian N, Akhavan Rezayat A, Hoseini SMR. Impact of Propranolol on Preventing Renal Dysfunction in Patients with Cirrhosis. Middle East J Dig Dis 2017;9:206-211. doi: 10.15171/mejdd.2017.75.

\section{INTRODUCTION}

Advanced cirrhosis is associated with poor clinical outcome and is a leading cause of death. ${ }^{1}$ Patients with liver cirrhosis regularly develop kidney dysfunction. In addition, kidney dysfunction, especially vasoconstriction of the renal arteries, plays an important role in the hepatorenal syndrome (HRS) and patients' prognosis. ${ }^{2}$ The impairment of renal function caused by marked vasoconstriction of the renal arteries is caused by complex changes in systemic hemodynamics. $^{2}$

Kidney failure caused by renal vascular constriction may persist weeks or even months before the development of clinical signs or before the rise in the 
level of serum urea and creatinine concentrations. ${ }^{3}$ As the disease progresses, the hemodynamic disturbances expand into the splanchnic and systemic circulatory beds. At end-stage liver disease, systemic vascular resistance is markedly decreased due to arteriolar vasodilatation induced by the release of mediators. ${ }^{4}$ In compensated phase, increases in plasma volume and cardiac output lead to the stability of the effective arterial volume and pressure. ${ }^{5}$ In advanced cirrhosis, however, the splanchnic vasodilatation is so extreme, which overwhelms this compensatory mechanism. ${ }^{6}$ Consequently, activation of endogenous vasoconstrictor mediators causes renal vasoconstriction and sodium and fluid retention. These events eventually lead to the evolution of ascites and renal dysfunction.?

Doppler renal ultrasonography is a simple and noninvasive diagnostic tool, which is widely used to study blood flow and arterial vascular resistance as parameters for vasoconstriction in liver cirrhosis. ${ }^{8}$ This method enables the accurate study of renal vascular hemodynamics, which has been validated in several pathological conditions. ${ }^{9}$ Doppler ultrasound measurement of the resistive index (RI) is used to quantify renovascular resistance in patients with cirrhosis before HRS development. ${ }^{10}$ It has also been found that abnormal raised impedance values have prognostic value in the identification of patients who are at greater risk for subsequent development of complications such as refractory ascites, HRS, and death. ${ }^{11}$

In patients with cirrhosis, early kidney failure or renal arteries vasoconstriction can be predicted by renal arterial RI measurement. ${ }^{12}$ Beta-blockers have been widely used for the prevention of variceal hemorrhage in patients with cirrhosis. But their efficacy has rarely been evaluated on renal hemodynamics of patients with cirrhosis..$^{13}$ Administering propranolol causes increased level of noradrenaline and, as a consequence, sodium and water retention. The other effect is reduction in plasma renin, angiotensin, and aldosterone levels ( $\beta 1$ blockade), which increases salt and water excretion. On the other hand, renal vasoconstriction due to $\beta 2$ blockade has been proposed as a mechanism by which $\beta$ blockers may impair renal blood flow (RBF) and glomerular filtration rate (GFR). However, the clinical significance of these changes and the impact on patients with cirrhosis remain to be elucidated. ${ }^{14}$

\section{MATERIALS AND METHODS}

The study was approved by the medical Ethics Committee of Mashhad university of medical sciences and informed consent was obtained from all participants. The patients with liver tumors, having an infection or gastrointestinal bleeding during the course of admission, hepatorenal syndrome, therapy with vasoactive drugs (including beta blockers and diuretics), significant concomitant disease, and co-therapy, which might interfere Doppler ultrasound parameters, and renal function were excluded from the study.

Doppler ultrasound measurements of the right interlobar renal artery and the left interlobar renal artery were obtained by a single investigator according to standard protocol and the mean value was calculated. The RI was calculated using the formula RI = (peak systolic velocity - end diastolic velocity)/ peak systolic velocity.

Laboratory results for liver and renal functions (serum bilirubin, albumin, prothrombin time, transaminases, cholinesterase, creatinine, urea nitrogen, and electrolytes) as well as a set of clinical parameters including arterial blood pressure, heart rate, age, sex, signs of hepatic encephalopathy, and finally the Child-Pugh score was collected to assess the severity of liver disease. The Model for End-Stage Liver Disease (MELD) score was calculated for all the patients according to the following formula: MELD score $=9.57 \times$ loge $[$ serum creatinine $(\mathrm{mg} / \mathrm{dL})]$ $+3.78 \times$ loge $[$ serum bilirubin $(\mathrm{mg} / \mathrm{dL})+11.20 \times \log \mathrm{e}$ $(\mathrm{INR})+6.43]$.

The standard protocol of incremental dosing of beta blockers was used to achieve the target heart rate. Propranolol was started at a dose of $20 \mathrm{mg}$ twice daily. The dose of propranolol was increased every 3 to 5 days as long as $25 \%$ of the resting heart rate was decreased or to the maximal dose of $320 \mathrm{mg} /$ day if the medication was well tolerated and the systolic blood pressure remained $\geq 90$ $\mathrm{mmHg}$. The second Doppler study of renal vasculature was done to measure RI by the same operator one month after continuous administration of target dose of propranolol.

The diagnosis of liver cirrhosis was based on clinical, laboratory, and radiological findings. Besides the standard hematological and biochemical tests, we also tested GFR $(\mathrm{mL} / \mathrm{min})$, and correct GFR $(\mathrm{GFR} \times 1.73 /$ body surface, $\mathrm{mL} / \mathrm{min})$. 
Table 1: Clinical characteristics and parameters of renal and hepatic function in patients with cirrhosis

\begin{tabular}{lc}
\hline Variables & Values \\
\hline Sex (male/female) & $21 / 9$ \\
\hline Age (years) & $51.97 \pm 11.56$ \\
\hline Ascites (yes/no) & $13 / 27$ \\
\hline Time from diagnosis of liver cirrhosis to study (year) & $6.09 \pm 4.81$ \\
\hline Serum Cr (mg/dL) & $1.09 \pm 0.24$ \\
\hline Etiology of liver cirrhosis, $\mathbf{n}(\%)$ & \\
\hline HBV infection & $13(47.2 \%)$ \\
\hline Cryptogenic & $6(16.7 \%)$ \\
\hline HCV infection & $5(16.7 \%)$ \\
\hline AIH & $4(11.1 \%)$ \\
\hline Wilson disease & $1(5.6 \%)$ \\
\hline PBC & $1(5.6 \%)$ \\
\hline Abbreviations: Cr, creatinine; HBV, hepatitis B virus; HCV, hepatitis C virus; AIH, \\
Auto-immune hepatitis; PBC, Primary biliary cirhosis.
\end{tabular}

Table 2: Resistive index and glomerular filtration rate in patients with cirrhosis before and after administering propranolol

\begin{tabular}{lcccc}
\hline Variables & $\mathbf{N}$ & Before & After & $\boldsymbol{p}$-value \\
\hline RI & & & & \\
\hline Compensated & 17 & $0.64 \pm 0.03$ & $0.64 \pm 0.02$ & 0.270 \\
\hline Decompensated & 13 & $0.72 \pm 0.02$ & $0.66 \pm 0.04$ & $<0.01$ \\
\hline Total & 30 & $0.67 \pm 0.04$ & $0.65 \pm 0.03$ & $<0.01$ \\
\hline GFR & & & \\
\hline Compensated & 17 & $72.80 \pm 21.49$ & $68.57 \pm 9.41$ & 0.333 \\
\hline Decompensated & 13 & $60.53 \pm 10.24$ & $74.02 \pm 10.10$ & $<0.01$ \\
\hline Total & 30 & $67.48 \pm 18.34$ & $70.93 \pm 9.93$ & 0.290 \\
\hline
\end{tabular}

GFR: glomerular filtration rate, RI: resistive index

\begin{tabular}{lccc}
$\begin{array}{c}\text { Table 3: Comparison of resistive index and glomerular filtration rate } \\
\text { between patients with compensated and decompensated cirrhosis } \\
\text { before and after administering propranolol }\end{array}$ & \\
\hline Variables & $\begin{array}{c}\text { Compensated } \\
(\mathbf{n}=\mathbf{1 7})\end{array}$ & $\begin{array}{c}\text { Decompensated } \\
(\mathbf{n}=\mathbf{1 3})\end{array}$ & p-value \\
\hline RI & $0.64 \pm 0.03$ & $0.72 \pm 0.02$ & $<0.001$ \\
\hline Before & $0.64 \pm 0.02$ & $0.66 \pm 0.04$ & 0.089 \\
\hline After & $-0.005 \pm 0.017$ & $-0.058 \pm 0.045$ & $<0.01$ \\
\hline$\Delta($ After-Before) & & & \\
\hline GFR & $72.80 \pm 21.49$ & $60.53 \pm 10.24$ & 0.068 \\
\hline Before & $68.57 \pm 9.41$ & $74.02 \pm 10.10$ & 0.139 \\
\hline After & $-4.226 \pm 17.440$ & $13.486 \pm 12.047$ & $<0.01$ \\
\hline$\Delta$ (After-Before) & & & \\
\hline GFR: glomerular filtration rate, RI: resistive index & & \\
\hline
\end{tabular}

GFR: glomerular filtration rate, RI: resistive index
Distribution of all continuous variables in this study was normal and they were reported as mean \pm standard deviations. Differences between the two groups were tested by using independent sample $t$ test for continuous data. The difference for GFR and RI variables before and after consumption of propranolol were tested by using paired sample $t$ test. Relations between continuous data were tested by using Pearson. All the data were analyzed using SPSS software version 11 (SPSS Inc., Chicago, IL, USA). $p<0.05$ was considered as statistically significant.

\section{RESULTS}

Thirty patients (21 men, 9 women) with liver cirrhosis, with the mean age of $51.97 \pm 11.56$ years (range $31-80$ years) were included in this study. Of them, 17 patients had compensated cirrhosis and 13 patients had decompensated condition. The main characteristics of the patients with liver cirrhosis in this study are demonstrated in table 1. Hepatitis B virus infection (HBV) $(n=13)$ was the most frequent etiology of cirrhosis. Other etiologies consist of hepatitis $\mathrm{C}$ virus infection $(\mathrm{HCV})(\mathrm{n}=5)$, cryptogenic $(\mathrm{n}=6)$, Wilson disease $(\mathrm{n}=1)$, and primary biliary cirrhosis $(\mathrm{n}=1)$.

As shown in table 2, in patients with cirrhosis, after administering propranolol, a significant decrease of RI was observed (from $0.67 \pm 0.04$ to $0.65 \pm 0.03 ; p<0.01$ ). However, there was no significant difference in the GFR before and after administering propranolol (from 67.48 \pm 18.34 to $70.93 \pm 9.93 ; p=0.290$ ). Pearson regression analysis showed no significant correlation between RI and GFR before $(\mathrm{r}=-0.084, p=0.659)$ and after $(\mathrm{r}=0.184$, $p=0.331)$ administering propranolol among the patients with cirrhosis. Independent sample t test showed that RI of patients with decompensated cirrhosis was significantly higher than compensated ones (compensated: $0.64 \pm 0.03$ vs. $0.72 \pm 0.02$ in decompensated patients; $p<0.001)$. However, there was no significant difference in the RI between the patients with compensated and decompensated cirrhosis after administering propranolol $(0.64 \pm 0.02$ vs. $0.66 \pm 0.04$ respectively; $p=0.089)$.

As shown in table 3 there were not any significant differences between the GFR of the patients with compensated and decompensated cirrhosis before $(72.80 \pm$ 21.49 vs. $60.53 \pm 10.24$ respectively; $p=0.068$ ) and after $(68.57 \pm 9.41$ vs. $74.02 \pm 10.10$ respectively; $p=0.139)$ administering propranolol. 
In our study, we found that administering propranolol was associated with marked significant changes in RI and GFR between the patients with compensated and decompensated cirrhosis (mean change: $-0.005 \pm 0.017$ vs. $-0.058 \pm 0.045 ; p<0.01$ for RI and $-4.226 \pm 17.440$ vs. $13.486 \pm 12.047 ; p<0.01$ for GFR in patients with compensated and decompensated cirrhosis, respectively).

\section{DISCUSSION}

In this study, after analyzing the results, RI in patients with decompensated cirrhosis was significantly higher than compensated ones. In the next step, the effect of propranolol in reducing the RI was reviewed and approved. This effect in patients with decompensated cirrhosis was significant, but in patients with compensated cirrhosis was not significant despite the reduction in RI. Also, there were significant differences between the effects of propranolol on the RI changes of the two mentioned groups. Our data support the previous findings that RI in patients with decompensated cirrhosis was significantly higher than compensated ones. In a study of 50 adult patients, with compensated and decompensated liver cirrhosis, mean values of renal arterial RI for the patients was higher than the 15 healthy control individuals.15 Furthermore, RI was higher in patients with decompensated cirrhosis than patients with compensated cirrhosis.

These results suggest that the degree of renal vasoconstriction alters with the severity of ascites. ${ }^{15}$ At the various stages of hepatic cirrhosis, there may be different degrees of renal arterial vasoconstriction, which can elicit to a decrease in renal blood flow, resulting in oliguria and anuresis. ${ }^{16}$ Ozkan and colleagues investigated 36 patients with decompensated cirrhosis, 39 patients with compensated cirrhosis, and 25 patients with normal kidney and liver functions. They found that RI was significantly higher in the decompensated group compared with the other groups. ${ }^{17}$ In the literature, three other studies can be found that evaluated RI measurement in patients with cirrhosis with and without ascites. These studies also revealed higher RI values in patients with cirrhosis and ascites compared with the ones without ascites..$^{9,18-19}$

Renal hemodynamic changes occur in early stages of cirrhosis before the development of ascites. However, as the liver disease progresses, these changes lead ultimately to severe cortical hypoperfusion. ${ }^{20}$ The development of portal hypertension is a probable promoter of increased renal vascular resistance, whereas liver function deterioration may be associated with the impaired tubular handling of sodium. ${ }^{21}$ The peripheral arterial vasodilation appears to be correlated with these renal changes by activation of vasoconstrictor systems after the development of arteriolar vasodilatation. $^{22}$

In the next step, the effect of propranolol in reducing the RI in patients with decompensated cirrhosis was significant, but in patients with compensated cirrhosis, despite the reduction in RI, was not significant. These results are in contradiction with the data that reported by Ozcan who showed after administering propranolol, RI decreased in the compensated patients but increased in the decompensated ones. There was a weak but statistically insignificant increase in the control individuals. ${ }^{17}$

Systemic and splanchnic hemodynamics, renal blood flow, and kidney function were evaluated in 13 patients with cirrhosis before and after oral acute and chronic administering $40 \mathrm{mg}$ propranolol. Kidney blood flow and vascular resistance did not alter significantly after acute administering propranolol and kidney function did not alter significantly after acute or chronic administering propranolol. The researchers conclude that propranolol does not change kidney function in cirrhotic patients with good physical condition. ${ }^{23}$ The renal effects of propranolol are complex based on their hemodynamic and neurohumoral actions. Propranolol can increase the level of noradrenaline, which in turn leads to sodium and water retention. As mentioned above, another consequence of treatment with propranolol is reduction in the levels of renin, angiotensin, and aldosterone, which increase the excretion of salt and water. ${ }^{13}$ In addition, kidney vasoconstriction caused by $\beta 2$ blockade has been proposed as a mechanism by which $\beta$ blockers may impair RBF and renal function. ${ }^{22}$

We did not find a significant correlation between RI values and GFR before and after administering propranolol among the patients with cirrhosis. The data about the relationship between RI and the renal function are controversial. Increased creatinine level is associated with concordance increase in RI. ${ }^{20}$ Renal artery RI may be useful for identifying patients at increased 
risk for developing kidney failure at an early stage. On the other hand, due to the paucity of available studies, there is no evidence that Doppler ultrasonography, by itself, can differentiate the patients with cirrhosis and impaired kidney function because of vasoconstriction, from the patients who have both vasoconstriction and intrinsic kidney damage. ${ }^{11}$

Renal vasoconstriction has been observed in several series of patients with cirrhosis due to increased RI. ${ }^{1}$ In patients with cirrhosis and refractory ascites, including those with a normal serum creatinine, increased RI seems to be correlated with a higher risk of subsequent impairment of kidney function. ${ }^{24}$ Similar results were observed when comparing cirrhosis patients with and without ascites and with the first signs of kidney failure. ${ }^{25}$

It has been hypothesized that peripheral arterial vasodilation is the main factor in the pathogenesis of functional kidney abnormalities in patients with cirrhosis. ${ }^{26}$ This alteration in peripheral vascular tonicity is thought to be related to local or systemic excess of vasodilators such as vasoactive intestinal peptide, prostacyclin, substance $\mathrm{P}$, and nitric oxide. ${ }^{27}$ The localization of arterial vasodilation, as well as its link with renal sodium retention, is also debated. ${ }^{28}$ According to recent clinical and experimental evidence, in patients with cirrhosis and ascites, reduction of arterial vascular resistance occurs mainly in the splanchnic area, whereas in the kidney vascular bed, arterial resistance is normal or even increased. ${ }^{8}$

\section{CONCLUSION}

The results of the present study demonstrate that propranolol reduces renal vascular RI in patients with cirrhosis. The response rates in patients with decompensated cirrhosis were significantly higher than the compensated type. Thus, non-selective beta blocker is a good treatment option for improvement of the renal hemodynamics in patients with cirrhosis, particularly decompensated ones. The effects of beta blockers on long-term protection of cirrhotic patients with renal dysfunction need more investigations to become an accepted recommendation.

\section{CONFLICT OF INTEREST}

The authors declare no conflict of interest related to this work.

\section{REFERENCES}

1. Asrani SK, Larson JJ, Yawn B, Therneau TM, Kim WR. Underestimation of liver-related mortality in the United States. Gastroenterology 2013;145:375-82 e2. doi:10.1053/j.gastro.2013.04.005.

2. Francoz C, Glotz D, Moreau R, Durand F. The evaluation of renal function and disease in patients with cirrhosis. $J$ Hepatol 2010;52:605-13. doi: 10.1016/j.jhep.2009.11.025.

3. Borse N, Sawant P, Gala B. Assessment of renal and hepatic hemodynamics in cirrhosis of liver. Indian J Gastroenterol 2002;21:213-5.

4. Sikarwar J, Muchhoria S, Singh R, Bhujade H, Ahirwar V. Study of resistive index in various stages of liver cirrhosis and its significance in calculating the risk for hepatorenal syndrome. J Evolution Med Dent Sci 2014;3:1195-206. doi: $10.14260 /$ jemds/2014/1968

5. Tyagi P, Sharma P, Sharma BC, Puri AS, Kumar A, Sarin SK. Prevention of hepatorenal syndrome in patients with cirrhosis and ascites: a pilot randomized control trial between pentoxifylline and placebo. Eur J Gastroenterol Hepatol 2011;23:210-7. doi: 10.1097/ MEG.0b013e3283435d76.

6. Arroyo V, Gines P, Gerbes AL, Dudley FJ, Gentilini P, Laffi G, et al. Definition and diagnostic criteria of refractory ascites and hepatorenal syndrome in cirrhosis. International Ascites Club. Hepatology 1996;23:164-76. doi: 10.1002/hep.510230122.

7. Cassinello C, Moreno E, Gozalo A, Ortuno B, Cuenca B, Solis-Herruzo JA. Effects of orthotopic liver transplantation on vasoactive systems and renal function in patients with advanced liver cirrhosis. Dig Dis Sci 2003;48:17986. doi:10.1023/A:1021763221337.

8. Maroto A, Gines P, Arroyo V, Gines A, Salo J, Claria $\mathrm{J}$, et al. Brachial and femoral artery blood flow in cirrhosis: relationship to kidney dysfunction. Hepatology 1993;17:788-93. doi: 10.1002/hep.1840170507.

9. Baik SK, Jee MG, Jeong PH, Kim JW, Ji SW, Kim HS, et al. Relationship of hemodynamic indices and prognosis in patients with liver cirrhosis. Korean J Intern Med 2004;19:165-70. doi:10.3904/kjim.2004.19.3.165.

10. Koda M, Murawaki Y, Kawasaki H. Renovascular resistance assessed by color Doppler ultrasonography in patients with chronic liver diseases. J Gastroenterol Hepatol 2000;15:1424-9. doi:10.1046/j.1440-1746.2000.02366.x.

11. Bardi A, Sapunar J, Oksenberg D, Poniachik J, Fernandez $\mathrm{M}$, Paolinelli P, et al. Intrarenal arterial doppler ultrasonography in cirrhotic patients with ascites, with and without hepatorenal syndrome. Rev Med Chil 2002;130:17380. doi:10.4067/S0034-98872002000200007.

12. Popov D, Krasteva R, Ivanova R, Mateva L, Krastev Z. Doppler parameters of hepatic and renal hemodynamics in patients with liver cirrhosis. Int $J$ Nephrol 2012;2012:961654. doi:10.1155/2012/961654.

13. Andreu V, Perello A, Moitinho E, Escorsell A, Garcia-Pa- 
gan JC, Bosch J, et al. Total effective vascular compliance in patients with cirrhosis. Effects of propranolol. J Hepatol 2002;36:356-61. doi:10.1016/S0168-8278(01)00300-2.

14. Aguilar-Olivos N, Motola-Kuba M, Candia R, Arrese M, Mendez-Sanchez N, Uribe M, et al. Hemodynamic effect of carvedilol vs. propranolol in cirrhotic patients: Systematic review and meta-analysis. Ann Hepatol 2014;13:420-8.

15. Wang Y, Liu LP, Bai WY, Wen SB, Dan HJ, Luan YY, et al. Renal haemodynamics in patients with liver cirrhosis assessed by colour ultrasonography. J Int Med Res 2011;39:249-55. doi:10.1177/147323001103900127.

16. Seo JI, Baik SK, Kim JW, Rhim DW, Park YS, Kim HS, et al. Renal Function Indices Predicting the Prognosis of Patients with Liver Cirrhosis. Korean J Hepatol 2001;7:140-6

17. Ozcan H, Aytac SK, Yagmurlu B, Erten A. Effect of propranolol on renal hemodynamics in patients with cirrhosis: assessment with Doppler US. Tani Girisim Radyol 2004;10:230-3.

18. Celebi H, Donder E, Celiker H. Renal blood flow detection with Doppler ultrasonography in patients with hepatic cirrhosis. Arch Intern Med 1997;157:564-6. doi:10.1001/archinte.1997.00440260132016.

19. Colle I, Moreau R, Pessione F, Rassiat E, Heller J, Chagneau $\mathrm{C}$, et al. Relationships between haemodynamic alterations and the development of ascites or refractory ascites in patients with cirrhosis. Eur $J$ Gastroenterol Hepatol 2001;13:251-6.

20. Rivolta R, Maggi A, Cazzaniga M, Castagnone D, Panzeri A, Solenghi D, et al. Reduction of renal cortical blood flow assessed by Doppler in cirrhotic patients with refractory ascites. Hepatology 1998;28:1235-40. doi: 10.1002/ hep. 510280510 .

21. Goyal S, Dixit VK, Jain AK, Shukla RC, Ghosh J, Kumar $\mathrm{V}$. Intrarenal resistance index (RI) as a predictor of early renal impairment in patients with liver cirrhosis. Trop Gastroenterol 2013;34:235-9.

22. Umgelter A, Reindl W, Franzen M, Lenhardt C, Huber W, Schmid RM. Renal resistive index and renal function before and after paracentesis in patients with hepatorenal syndrome and tense ascites. Intensive Care Med 2009;35:152-6. doi:10.1007/s00134-008-1253-y.

23. Bataille C, Bercoff E, Pariente EA, Valla D, Lebrec D. Effects of propranolol on renal blood flow and renal function in patients with cirrhosis. Gastroenterology 1984;86:129-33.

24. Kastelan S, Ljubicic N, Kastelan Z, Ostojic R, Uravic $\mathrm{M}$. The role of duplex-doppler ultrasonography in the diagnosis of renal dysfunction and hepatorenal syndrome in patients with liver cirrhosis. Hepatogastroenterology 2004;51:1408-12.

25. Rendon Unceta P, Macias Rodriguez MA, Guillen Mariscal P, Tejada Cabrera M, Martinez Sierra MC, Martin Herrera L. Renal Doppler ultrasonography and its rela- tionship with the renal function in patients with liver cirrhosis. Med Clin (Barc) 2001;116:561-4. doi: 10.1016/ S0025-7753(01)71906-9.

26. Schrier RW, Arroyo V, Bernardi M, Epstein M, Henriksen JH, Rodes J. Peripheral arterial vasodilation hypothesis: a proposal for the initiation of renal sodium and water retention in cirrhosis. Hepatology 1988;8:1151-7. doi: 10.1002/hep.1840080532.

27. Bosch J, Gines P, Arroyo V, Navasa M, Rodés J. Hepatic and systemic hemodynamics and the neurohumoral systems in cirrhosis. In: Epstein M, ed. The kidney in liver disease 3rd ed Baltimore: Williams \& Wilkins 1988:286-305.

28. Fernandez-Seara J, Prieto J, Quiroga J, Zozaya JM, Cobos MA, Rodriguez-Eire JL, et al. Systemic and regional hemodynamics in patients with liver cirrhosis and ascites with and without functional renal failure. Gastroenterology 1989;97:1304-12. 